Urologe $2017 \cdot 56: 977-978$

DOI 10.1007/s00120-017-0456-7

Online publiziert: 10. Juli 2017

(c) Springer Medizin Verlag GmbH 2017

CrossMark

\author{
Axel Schroeder ${ }^{1}$ Tilman Kälble ${ }^{2}$ Wolfgang Bühmann ${ }^{3}$ \\ ${ }^{1}$ Neumünster, Deutschland \\ ${ }^{2}$ Klinik für Urologie und Kinderurologie, Klinikum Fulda gAG, Fulda, Deutschland \\ ${ }^{3}$ Wenningstedt-Braderup, Deutschland
}

\title{
Berufspolitik - Brücke zwischen Wissenschaft und ärztlichem Alltag
}

Liebe Kolleginnen und Kollegen,

turnusgemäß widmet sich "Der Urologe" im August-Heft dem Leitthema der Berufspolitik unter der Federführung des Berufsverbandes der Deutschen Urologen in Zusammenarbeit mit der Deutschen Gesellschaft für Urologie.

Warum eigentlich? Berichten wir doch monatlich im Vereinsorgan mit BDUJournal zur aktuellen Berufspolitik. In der August-Ausgabe des Urologen stellen wir Ihnen einige wichtige Themen aus der Berufs- und Standespolitik des Jahres 2017 vor, die für uns Urologinnen und Urologen in Klinik und Praxis in unserem täglichen Arbeiten von Bedeutung sind, mit denen wir uns als BDU und DGU berufspolitisch bzw. standespolitisch beschäftigen.

Der Arzt als freier Beruf in Klinik und Praxis stand noch nie in solch einem Maße zur Disposition wie in den letzten Jahren. Ökonomie, überbordende Bürokratie und Administration mischen sich immer deutlicher in das unabhängige Arzt-Patientenverhältnis ein.

Heute bedarf es hochkompetenter ärztlicher Profis aus Klinik und Praxis, diese Interessen zum Wohle unserer Patienten zu vertreten. Aktuell wird Medizin leider nicht mehr dem Bedarf der Kranken, sondern dem Bedarf der Ökonomen nach Einnahmen und Ausgaben angepasst - welch ein Armutszeugnis für eine hochkultivierte und wohlhabende Gesellschaft!

So spannen wir den Bogen unserer urologisch-berufspolitischen Arbeit, um transparent zu machen, wie der durch fach- und sachferne Akteure verursachte Schaden begrenzt werden kann.

Andreas Gassen, gerade bestätigter Vorstandsvorsitzender der Kassenärztlichen Bundesvereinigung, eröffnet den Reigen mit seiner Betrachtung zum „Vertragsarzt 2020“: Die Medizin, die Technik, die politischen Konstanten sind in permanenter Bewegung und verändern sich. Seit einigen Jahren nimmt die Dynamik darin erheblich zu und hat ihre deutlichen Auswirkungen auf die einzelne Vertragsarztpraxis. Wenn die Vertragsärzte sich nicht selbst bewegen, werden sie bewegt. Das sehen wir an Themen wie Bereitschaftsdienst und Notfallversorgung, Digitalisierung, Telemedizin und auch am Entstehen neuer Gesundheitsberufe.

Lars Lindemann, Geschäftsführer des Spitzenverbandes Fachärzte Deutschlands e.V. (SpiFa), wirft einen Ausblick auf die gesundheitspolitischen Positionen der Bewerber um die Wählergunst:

Die Bundestagswahl steht kurz bevor und wie in jedem Bundestagswahlkampf gilt, dass man mit Gesundheitspolitik keine Wahl gewinnt, wohl aber damit verlieren kann. So wird es dann auch dieses Mal in der Auseinandersetzung der Parteien wieder um gesundheitspolitische Grundsatzfragen gehen, aber man ist dabei gut beraten, auch auf die Details zu achten.

BDU-Präsident Axel Schroeder und seine Co-Autoren aus Berufsverband und DGU präsentieren die Forderungen zur Ambulanten Spezialfachärztlichen Versorgung (ASV; $\$ 116 \mathrm{~b}$ neu), die alsbald auch Veränderungen in der zukünftigen ambulanten Uroonkologie bringen wird, um dieses bürokratische Schwergewicht für Urologen in Klinik und Praxis möglichst lebbar zu gestalten.

Stephan Roth, Klinikdirektor in Wuppertal und Lehrstuhlinhaber in Witten/ Herdecke, gestaltet mit seinen $\mathrm{Co}-\mathrm{Au}-$ toren die Ausbildung von „Assistenten/ Innen für Urologische Kontinenztherapie" mit dem Ziel, nicht-ärztliche Mitarbeiter/-innen (NÄPA) in urologischen Praxen und Kliniken zu qualifizieren, bestimmte Aufgaben zum Management der Harninkontinenz zu übernehmen und damit u. a. Ärzte im Rahmen der Delegation in Praxis und Klinik zu unterstützen und auch zu entlasten. Diese Fortbildungsreihe wird Grundlage einer urologischen curriculären Fortbildung für nicht-ärztliche Praxisassistenten sein - z. B. eine Voraussetzung, um die Förderung unseres Assistenzpersonals und Teilnahme am EBM-Kapitel 38 im Zusammenhang mit dem Kooperationsvertrag mit Alten- und Pflegeheimen nach $\$ 119$ b zu ermöglichen - seit dem 01.07.2017 auch für die häusliche Versorgung.

In dem Beitrag „Urologische Geriatrie - zunehmende Bedeutung in der urologischen Versorgung" beleuchtet Andreas Wiedemann, urologischer Chefarzt und Lehrstuhlinhaber für Geriatrie in Witten/ Herdecke, die Auswirkungen der demografischen Patientenentwicklung in der Urologie mit der zukünftig höchsten $\mathrm{Zu}$ nahme an Versorgungsbedarf unter allen ärztlichen Fachgruppen.

Die „Novellierung der Musterweiterbildungsordnung Urologie - Zukunftssicherung unseres Fachgebiets?" rückt Oli- 
ver W. Hakenberg, Lehrstuhlinhaber für Urologie in Rostock und 2. DGU-Vizepräsident, in den Blickpunkt, um die Probleme zu verdeutlichen, die geforderten Weiterbildungsinhalte für die künftige urologische Facharztreife in vertretbarer Zeit vermitteln zu können. Diese Novelle hat damit erhebliche berufspolitische Brisanz.

Damit auch die aktiven Helfer nicht völlig in Vergessenheit geraten, erfahren wir von Hans-Friedrich Spies, Internist aus Wiesbaden und Vorstandsmitglied des SpiFa, den Sachstand zur seit Jahren strittigen Dauerbaustelle „GOÄ“, der seit 21 Jahren inhaltlich und merkantil unveränderten Gebührenordnung für Ärzte - an dieser werden wir ermessen können, was unsere Arbeit der Gesellschaft wirklich wert ist: „Die neue GOÄ und ihre Fallstricke“.

Den Abschluss bildet Christian Wülfing, Chefarzt der Urologischen Klinik in Hamburg-Altona und DGU-Pressesprecher, mit dem Zukunftsthema „Imagepflege für Deutschlands Urologen - vom Strategieworkshop bis zur Bildung der ,Marke Urologie“, um die deutlichen Mängel der Urologie in der öffentlichen Wahrnehmung auszugleichen - Urologen behandeln den unerfüllten Kinderwunsch genauso wie die potentiell lebensbegrenzenden Krankheiten beider Geschlechter am anderen Ende der Lebensskala, getreu dem diesjährigen Kongressmotto: „Urologie - Für Alle. Für Jeden. Für Uns.“

So hoffen wir, einen gehaltvollen, ernsthaften, dennoch wohlschmeckenden berufspolitischen Cocktail für Sie gemixt zu haben, der Ihnen hilft, die Freude an unserem Beruf zu erhalten.

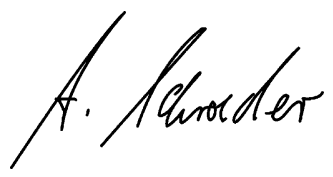

Axel Schroeder BDU-Präsident

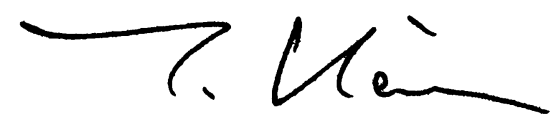

Tilmann Kälble

DGU-Präsident

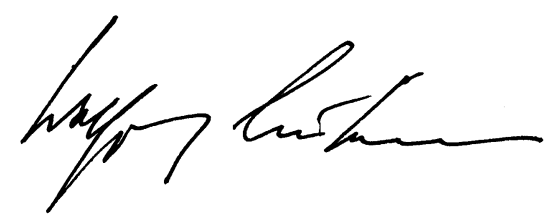

Wolfgang Bühmann

BDU-Schriftleiter

\section{Korrespondenzadresse}

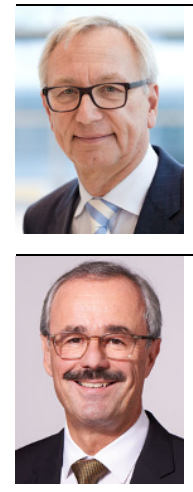

Dr. A. Schroeder

Haart 87-89, 24534 Neu-

münster, Deutschland

praesident@bdu-urologie.de

\section{Prof. Dr. T. Kälble}

Klinik für Urologie und

Kinderurologie, Klinikum Fulda gAG

Pacelliallee 4, 36043 Fulda,

Deutschland

t.kaelble.urologie@klinikumfulda.de

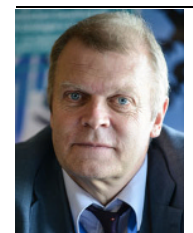

Dr. W. Bühmann

Osetal 7, 25996 Wenningstedt-Braderup,

Deutschland

buehmann@bdu-urologie.de

Interessenkonflikt. A. Schroeder, T. Kälble und W. Bühmann geben an, dass kein Interessenkonflikt besteht. 